\title{
Metabolic profiling of human brain metastases using in vivo proton MR spectroscopy at 3T
}

\author{
Torill E Sjøbakk*1, Roar Johansen ${ }^{2}$, Tone F Bathen ${ }^{3}$, Ursula Sonnewald ${ }^{1}$, \\ Kjell A Kvistad ${ }^{4}$, Steinar Lundgren ${ }^{2,5}$ and Ingrid S Gribbestad ${ }^{3}$
}

Address: ${ }^{1}$ Department of Neuroscience, Norwegian University of Science and Technology (NTNU), Norway, ${ }^{2}$ Department of Cancer Research and Molecular Medicine, NTNU, Trondheim, Norway, ${ }^{3}$ Department of Circulation and Medical Imaging, NTNU, Trondheim, Norway, ${ }^{4}$ Department of Radiology, St. Olavs University Hospital, Trondheim, Norway and ${ }^{5}$ Department of Oncology, St. Olavs University Hospital, Trondheim, Norway

Email: Torill E Sjøbakk* - Torill.Sjobakk@ntnu.no; Roar Johansen - roar.johansen@ntnu.no; Tone F Bathen - tone.f.bathen@ntnu.no; Ursula Sonnewald - Ursula.Sonnewald@ntnu.no; Kjell A Kvistad - Kjell.Arne.Kvistad@stolav.no;

Steinar Lundgren - Steinar.Lundgren@stolav.no; Ingrid S Gribbestad - ingrid.s.gribbestad@ntnu.no

* Corresponding author

Published: 27 July 2007

BMC Cancer 2007, 7:14| doi:|0.||86/|47|-2407-7-|4|
Received: 20 March 2007

Accepted: 27 July 2007

This article is available from: http://www.biomedcentral.com/I47I-2407/7//4I

(C) 2007 Sjøbakk et al; licensee BioMed Central Ltd.

This is an Open Access article distributed under the terms of the Creative Commons Attribution License (http://creativecommons.org/licenses/by/2.0), which permits unrestricted use, distribution, and reproduction in any medium, provided the original work is properly cited.

\begin{abstract}
Background: Metastases to the central nervous system from different primary cancers are an oncologic challenge as the overall prognosis for these patients is generally poor. The incidence of brain metastases varies with type of primary cancer and is probably increasing due to improved therapies of extracranial metastases prolonging patient's overall survival and thereby time for brain metastases to develop. In addition, the greater access to improved neuroimaging techniques can provide earlier diagnosis. The aim of this study was to investigate the feasibility of using proton magnetic resonance spectroscopy (MRS) and multivariate analyses to characterize brain metastases originating from different primary cancers, to assess changes in spectra during radiation treatment and to correlate the spectra to clinical outcome after treatment.

Methods: Patients $(n=26)$ with brain metastases were examined using single voxel MRS at a $3 T$ clinical MR system. Five patients were excluded due to poor spectral quality. The spectra were obtained before start $(n=21$ patients), immediately after $(n=6$ patients) and two months after end of treatment ( $n=4$ patients). Principal component analysis (PCA) and partial least square regression analysis (PLS) were applied in order to identify clustering of spectra due to origin of metastases and to relate clinical outcome (survival) of the patients to spectral data from the first MR examination.

Results: The PCA results indicated that brain metastases from primary lung and breast cancer were separated into two clusters, while the metastases from malignant melanomas showed no uniformity. The PLS analysis showed a significant correlation between MR spectral data and survival five months after MRS before start of treatment.

Conclusion: MRS determined metabolic profiles analysed by PCA and PLS might give valuable clinical information when planning and evaluating the treatment of brain metastases, and also when deciding to terminate further therapies.
\end{abstract}




\section{Background}

Brain metastases are a common oncologic challenge as approximately 15-20\% of all cancer patients develop central nervous system metastases. They are the dominating type of brain tumor and are most frequently caused by haematogenous spread from a primary cancer in lung, breast, skin or colon [1]. Survival varies with type and grade of primary cancer, age at diagnosis as well as extent of proliferation of the primary cancer [2]. The overall prognosis of survival for these patients is generally poor and the management of the disease is a significant challenge.

A major goal in oncology treatment is to give each patient an individualized therapeutic regime. In addition to diagnosis, the patient's pre-treatment performance status described by Karnofsky performance score (KPS) and the recursive partitioning analysis (RPA) is evaluated [3]. The standard treatment is whole-brain-radiation-therapy (WBRT) alone or after surgery which may prolong life from one to nine months [4]. Radiation therapy is the most common treatment strategy since more than $70 \%$ of the patients have multiple metastases at the time of diagnosis [5]. Chemotherapy may also be used in selected patients. To decide between different treatment options various prognostic factors such as age, performance status, numbers of brain metastases and type of primary cancer and extent of extracranial metastases activity are evaluated. Some patients get their brain metastases detected before the primary cancer and a non-invasive identification of type of metastases would be of importance for further treatment [6]. In order to optimize and avoid ineffective treatment there are also a need for early evaluation of response. The diagnosis and planning of brain tumor treatment have become more advanced due to improved diagnostic neuroimaging tools. One of these is in vivo magnetic resonance spectroscopy (MRS) which provides additional information for classifying most brain tumor types and grades [7-9].

Proton $\left({ }^{1} \mathrm{H}\right)$ in vivo MRS can be used to quantify metabolites and monitor response to therapy in brain tumors, thereby allowing non-invasive monitoring of tumor biochemistry [7]. Previous studies have used 1.5T systems for classification and characterisations of brain tumors. Recent clinical instrument operating at 3T has improved spectral quality related to resolution and signal-to-noiseratio [10-12]. The main metabolites in proton spectra of normal brain tissue are $\mathrm{N}$-acetyl aspartate (NAA), creatine (Cr) and choline-containing compounds (tCho) at 2.0, 3.0 and $3.2 \mathrm{ppm}$, respectively. In brain malignancies lipid signals at 1.3 (methylene) and 0.9 ppm (methyl) appear when short echo times are used $[8,13]$. Previous studies have addressed metabolic changes in primary brain tumors occurring after radiation therapy [14-16]. The reports from different groups are not conclusive but reduction in tCho appears to be a reliable marker for treatment response. Among the parameters being predictive for poorer outcome in certain patient sub-groups were higher tCho/Cr and tCho/NAA ratios and higher lipid signals and lower Cr/NAA ratio [17]. Less is known about brain metastases and the objectives of this study were to characterize brain metastases originating from different primary cancers, to assess changes in spectra during radiation treatment and to correlate the spectra to clinical outcome for the patients after treatment. Our hypothesis is that in vivo MRS from different brain metastases analysed by multivariate analyses distinguish metastases originating from different primary tumors, and also that MR spectra can indicate clinical outcome for these patients.

\section{Methods \\ Patients}

A total of 26 consecutive patients ( 18 women and 8 men) with brain metastases from primary cancers such as breast $(n=9)$, lung $(n=9)$, malignant melanoma $(n=4)$, colon $(n=3)$ and kidney $(n=1)$ were enrolled in this study. All patients gave a written informed consent and the study was approved by the local ethics board; Central Norway medical reach ethics committee. After contrast enhanced MRI, metastases larger than $10 \mathrm{~mm}$ in diameter were chosen for MRS. The quality evaluation of each MR spectrum, led to the exclusion of five of the 26 enrolled patients (one from each primary cancer group). A description of the 21 remaining patients is given in Table 1 . Six patients came to a second examination immediately after completed radiation treatment and four patients to a third MRI/MRS examination after additional two months. The same size of volume of interest (VOI) was used in each of the repeated examinations. The patients' performance status (KPS and RPA) was determined before start of treatment.

\section{In vivo $M R$ spectroscopy}

MR imaging and spectroscopy examinations were performed using a 3T clinical MR system (Philips Intera, Best, The Netherlands) equipped with a transmit-receive head coil. The MRI protocol consisted of conventional $\mathrm{T}_{1}-$ and $\mathrm{T}_{2}$ - weighted images before and after an intravenous injection of $0.2 \mathrm{ml}$ Gadodiamide/ $\mathrm{kg}$ body weight (Omnis$\operatorname{can}^{\mathrm{TM}}$, GE Healthcare). Single voxel ${ }^{1} \mathrm{H}$ MRS was performed after contrast injection on a VOI localized within the metastases using the point resolved spectroscopy (PRESS) pulse sequence, repetition time (TR) of $2000 \mathrm{~ms}$ and echo time (TE) of 32/33 ms. When only one metastasis (>10 mm) was detected, a second MRS acquisition was performed of the same volume using TE $=144 \mathrm{~ms}$ (18 patients). If two or more large metastases were identified by the MRI, a second spectrum was obtained using the short TE in one of the other tumors ( 7 patients). The VOI was $15 \times 15 \times 15$ or $10 \times 10 \times 10 \mathrm{~mm}^{3}$, depending on the 
Table I: Patient characteristic. Treatment and clinical outcome data for all patients with approved MR spectra.

\begin{tabular}{|c|c|c|c|c|c|c|c|c|c|c|}
\hline $\begin{array}{l}\text { Patient } \\
\text { no. }\end{array}$ & Sex, Age & $\begin{array}{l}\text { Primary } \\
\text { cancer }\end{array}$ & $\begin{array}{l}\text { Brain } \\
\text { mets }\end{array}$ & $\begin{array}{l}\text { Mets other } \\
\text { sites than brain }\end{array}$ & KPS & RPA & $\begin{array}{l}\text { Survival } \\
\text { (months) }\end{array}$ & Treatment & $\begin{array}{l}\text { Time: diagnosis- } \\
\text { I.MRS (weeks) }\end{array}$ & $\begin{array}{c}\text { Spectral data TE, } \\
\text { VOII }^{1,2}\end{array}$ \\
\hline $\mathbf{I}$ & $\mathrm{F}, 52$ & Breast & Single & Brain only & 90 & 1 & $13+$ & $S+3 G y \times 10$ & 5 & $32,|44|$ \\
\hline 2 & M, 44 & Lung & Single & Brain only* & 90 & 1 & $10+$ & $\mathrm{S}$ & 1 & 33,1442 \\
\hline $3^{a}$ & $\mathrm{~F}, 47$ & Lung & Multiple & Brain only & 90 & I & $7+$ & $\mathrm{C}$ & 0 & $32,|44|$ \\
\hline $4^{a}$ & $\mathrm{~F}, 45$ & Breast & Multiple & Mediastinum & 100 & 2 & $15+$ & $S+3 G y \times 10$ & 1 & 33,1442 \\
\hline $5^{a}$ & $F, 48$ & Breast & Multiple & Skeleton* & 100 & 2 & $5+$ & $3 G y \times 10$ & 0 & $33^{2}$ \\
\hline 6 & $\mathrm{~F}, 57$ & Lung & Multiple & $\begin{array}{l}\text { Intestinal, } \\
\text { jejunum }\end{array}$ & 100 & 2 & 7 & $3 G y \times 10$ & 4 & $32,|44|$ \\
\hline 7 & $M, 63$ & Lung & Multiple & Lung, skeleton & 100 & 2 & 6 & $3 G y \times 10$ & 2 & $32,|44|$ \\
\hline 8 & $M, 62$ & Lung & Single & Skeleton & 90 & 2 & 4.5 & $S+3 G y \times 10$ & 4 & 33,1442 \\
\hline 9 & $M, 70$ & Mal. mel. & Multiple & Lung, subcutan* & 90 & 2 & 2 & $S+3 G y \times 10$ & 3 & 33 in $2 \mathrm{VOI}^{2}$ \\
\hline 10 & $\mathrm{~F}, 36$ & Breast & Multiple & Liver, skeleton & 80 & 2 & 9 & 4Gyx5 (GK) & 1 & $32,|44|$ \\
\hline I I & $\mathrm{F}, 47$ & Breast & Multiple & Liver & 80 & 2 & 10 & $3 \mathrm{Gyx} 10$ & 2 & $32,|44|$ \\
\hline $12^{\mathrm{b}}$ & $F, 66$ & Breast & Multiple & Brain only & 80 & 2 & 2 & $3 G y \times 10$ & 3 & 33 in $2 \mathrm{VOI}^{2}$ \\
\hline 13 & M, 70 & Colon & Single & Liver, lung & 80 & 2 & 6 & $S+4 G y \times 5$ & 17 & $33,|44|$ \\
\hline $14^{b}$ & $\mathrm{~F}, 80$ & Lung & Multiple & Brain only & 80 & 2 & 0.75 & 4Gyx5 & 1 & 33 in $2 \mathrm{VOI}^{2}$ \\
\hline $15^{a}$ & F, 7I & Lung & Multiple & Brain only & 80 & 2 & 3 & $3 G y \times 10$ & 4 & 33 in $2 \mathrm{VOI}^{2}$ \\
\hline 16 & $\mathrm{~F}, 72$ & Breast & Multiple & Liver, skeleton & 60 & 3 & 0.5 & 4Gyx5 & 2 & 33,1442 \\
\hline 17 & $M, 69$ & Colon & Single & Liver, lung & 60 & 3 & 6 & $S+3 G y x 10$ & 5 & $32,|44|$ \\
\hline $18^{\mathrm{a}}$ & $\mathrm{F}, 53$ & Lung & Multiple & Brain only* & 60 & 3 & $\mathrm{II}+$ & $4 G y \times 5$ & 2 & 33 in $2 \mathrm{VOI}^{2}$ \\
\hline 19 & M, 56 & Mal. mel. & Multiple & Epigastrium & 60 & 3 & 1 & No Treatment & 2 & $33^{2}$ and $32^{\prime}$ \\
\hline 20 & $\mathrm{~F}, 63$ & Breast & Single & $\begin{array}{l}\text { Lung, skeleton, } \\
\text { lymph node }\end{array}$ & 50 & 3 & 0.5 & No treatment & 0 & $33,144^{2}$ \\
\hline 21 & F, 7I & Mal. mel. & Single & Lung, liver & 50 & 3 & 1.5 & $3 G y x 10$ & 8 & $32, \mid 44^{\prime}$ \\
\hline
\end{tabular}

$\mathrm{F}=$ female, $\mathrm{M}=$ male, mets = metastases, a: three MRS follow-up examinations, b: two follow-up MRS examinations, $*$ : brain metastases detected before primary cancer, KPS = Karnofsky Performance Score, RPA = Recursive Partitioning Analysis classification, TE = echo time, VOI',2 = volume of interest, ': voxel size: $15 \times 15 \times 15 \mathrm{~mm}^{3}, 2: 10 \times 10 \times 10 \mathrm{~mm}^{3}$. Mal. mel. = malignant melanoma, GK = gamma knife, $\mathrm{S}=\mathrm{Brain}$ surgery and histopathologic verification, $\mathrm{C}=$ Chemotherapy.

size of the actual metastasis, and all localizations were verified by an experienced neuroradiologist. Bandwidth, number of points and sampling interval were $2000 \mathrm{~Hz} /$ $1024 / 0.5 \mathrm{~ms}$. Each spectrum was obtained as an average of 192 measurements, giving an acquisition time of 7 minutes. Shimming (automatic) prolonged the acquisition time with about three minutes. A routine water unsuppressed spectrum (16 measurements) obtained at each examination was used to evaluate the spectrum quality. A total of 33 spectra with short and 18 spectra with long TE were obtained from brain metastases during the MRS examinations. The spectroscopic data were postprocessed using jMRUI [18]. The FIDs were zero-filled (2 $\mathrm{K})$ and a Lorentzian filter $(2 \mathrm{~Hz})$ was applied before Fast Fourier Transformation (FFT). The residual peak of the water signal was suppressed using Hankel Lanczos Singular Values Decomposition Filter (HLSVD)[18].

\section{Data analyses}

The spectral quality was evaluated by applying the algorithm AMARES to estimate the linewidth (full-width-halfmaximum, FWHM) of the water peak signal in the corresponding water unsuppressed spectra. A mean FWHM \pm SD of water peaks in all spectra was calculated $(9.2 \pm 2.1$ $\mathrm{Hz}$ ). The mean value with the corresponding positive $99 \%$ CI $(2.1 \mathrm{~Hz})$ was chosen as quality criteria. All spectra with a higher FWHM than $11.3 \mathrm{~Hz}$ were excluded from further data analyses. Spectral data were transferred to the soft- ware program Unscrambler (CAMO) as ASCII-files, Baseline offset was adjusted and the lipid signal at $1.3 \mathrm{ppm}$ was used as a chemical shift reference. The spectra were mean normalised and examined using principal component analysis (PCA) and partial least square regression analysis (PLS). Only the chemical shift region including the resonances from lipids to tCho compounds $(0.70-$ $3.45 \mathrm{ppm} / 365$ spectral points) was investigated. Mean spectra for each primary cancer group were made to illustrate the spectral variations.

PCA was applied in order to identify clustering of spectra due to origin of metastases based on examination of score plots and loading profiles. The PCA model compresses or simplifies high-dimensional data by finding a linear combination of the original variables so the variance is maximized and new uncorrelated variables, principal components (PC) are created. To avoid that the model described too much of the variation in the data i.e. noise, the number of PCs to retain in the model was kept as low as possible. PCA was performed with full cross validation and mean centering.

The PLS was applied in order to relate clinical outcome of the patients (survival or not at five months after first MR examination) to the spectral data (obtained at first MR examination). PLS is the regression extension of the PCA and the data are reduced into PLS factors which explain 
most of the variation in both predictors (MR spectra) and responses (clinical parameters). The number of PCs to remain in the model was determined by finding the PC where the total residual y-variance and root mean square error of prediction (RMSEP) were minimized. For the PLS analysis the test set method was used as validation method. The test samples were selected in advance by using the Kennard Stones algorithm for splitting data sets into two subsets [19], resulting in 14 samples for calibration and 7 samples for testing. The significance of the estimated correlation factors between measured and predicted y-variables was ascertained by using the Pearson correlation test (two-tailed).

\section{Results}

Of the 33 short echo time spectra obtained from the enrolled 26 patients, six spectra were excluded due to a
FWHM of the water signal larger than $11.3 \mathrm{~Hz}$, likewise four of the eighteen spectra obtained at TE 144, leaving 27 spectra with short and 14 with long echo time for further analyses.

Spectra from two distinct metastases were obtained in six patients (Table 1). Typical axial T1 weighted contrast enhanced MR images with corresponding in vivo spectra of metastases in two breast cancer patients are given in Fig. 1. Mean spectra (short TE) $\pm 95 \%$ CI of the four different primary cancer groups are shown in Fig. 2 . The lipid signals at 1.3 and $0.9 \mathrm{ppm}$ were the dominating peaks in the majority of the spectra in all groups. $\mathrm{Cr}$, tCho and a broad peak around $2.0-2.2 \mathrm{ppm}$ were also detected in 17, 20 and 22 of the 27 spectra, respectively.
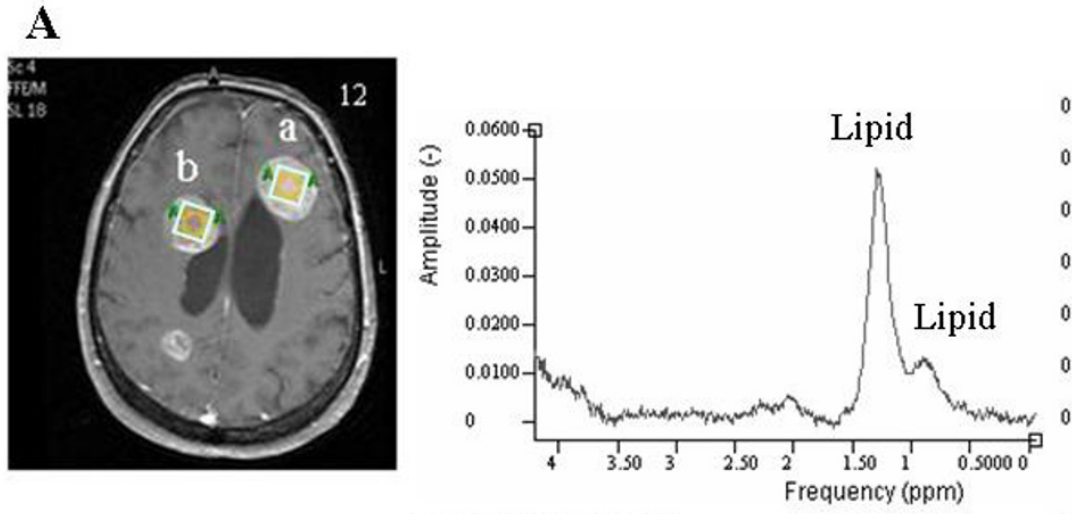

\section{VOI (a) TE 33}
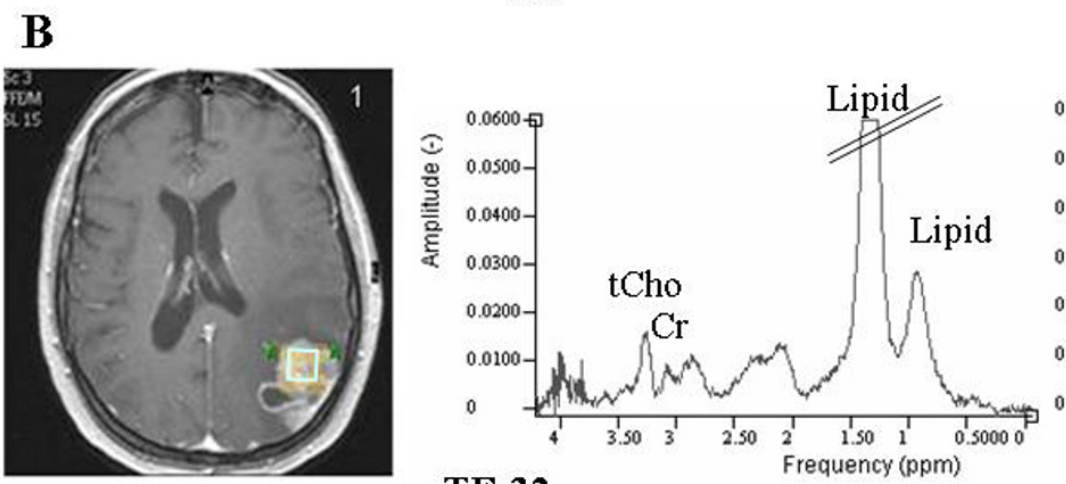

TE 32

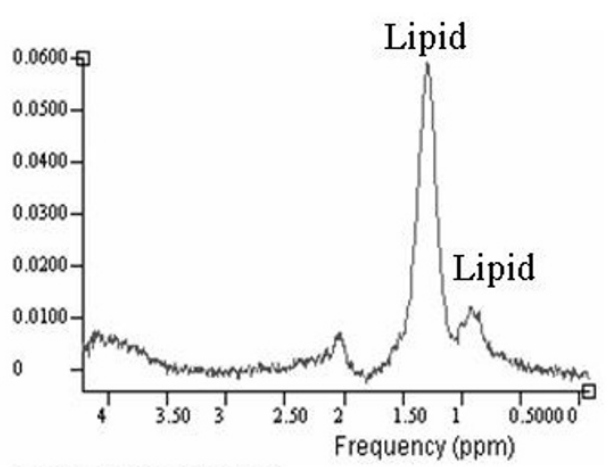

VOI (b) TE 33 
The score plot from PCA of the 27 short echo time spectra obtained before start of treatment is presented in Fig. $3 \mathrm{a}$. This plot indicates that brain metastases from primary lung and breast cancer tend to cluster, while the metastases from malignant melanomas show no uniformity. The two spectra of metastases from colon cancer are found in the lower right quadrant of the score plot. The separation of breast and lung metastases is based on PC1 and PC2, which account for more than $83 \%$ of the total variation of the spectra. Samples with high score for PC1 are characterized by high lipid signal and no other metabolites, as described by the loading profile of PC1 (Fig. 3b).

Figure 4 gives a score plot of PC1 versus PC2 from the PLS relating spectra of untreated metastases to survival five months after treatment. The first two PCs explained totally $85 \%$ of the x-variables (the spectral data) and 53 $\%$ of the $y$-variable (survival). The correlation factor between measured and predicted survival were 0.73 ( $\mathrm{p}<$ $0.01)$ for the calibration run and $0.76(\mathrm{p}<0.05)$ for the corresponding validation (seven test samples). The spectra of patients who survived five months after first MRS (marked as circles) are clustered on the right side of the score plot, while the spectra of patients who survived less than five months (cross marks) are clustered in the opposite side.

Follow-up MRS examinations where completed only for four of the included patients, since some got a complete surgical resection of the metastasis, a few refused further examinations and some died shortly after the first or second examination (Table 1). Two examples of the follow-

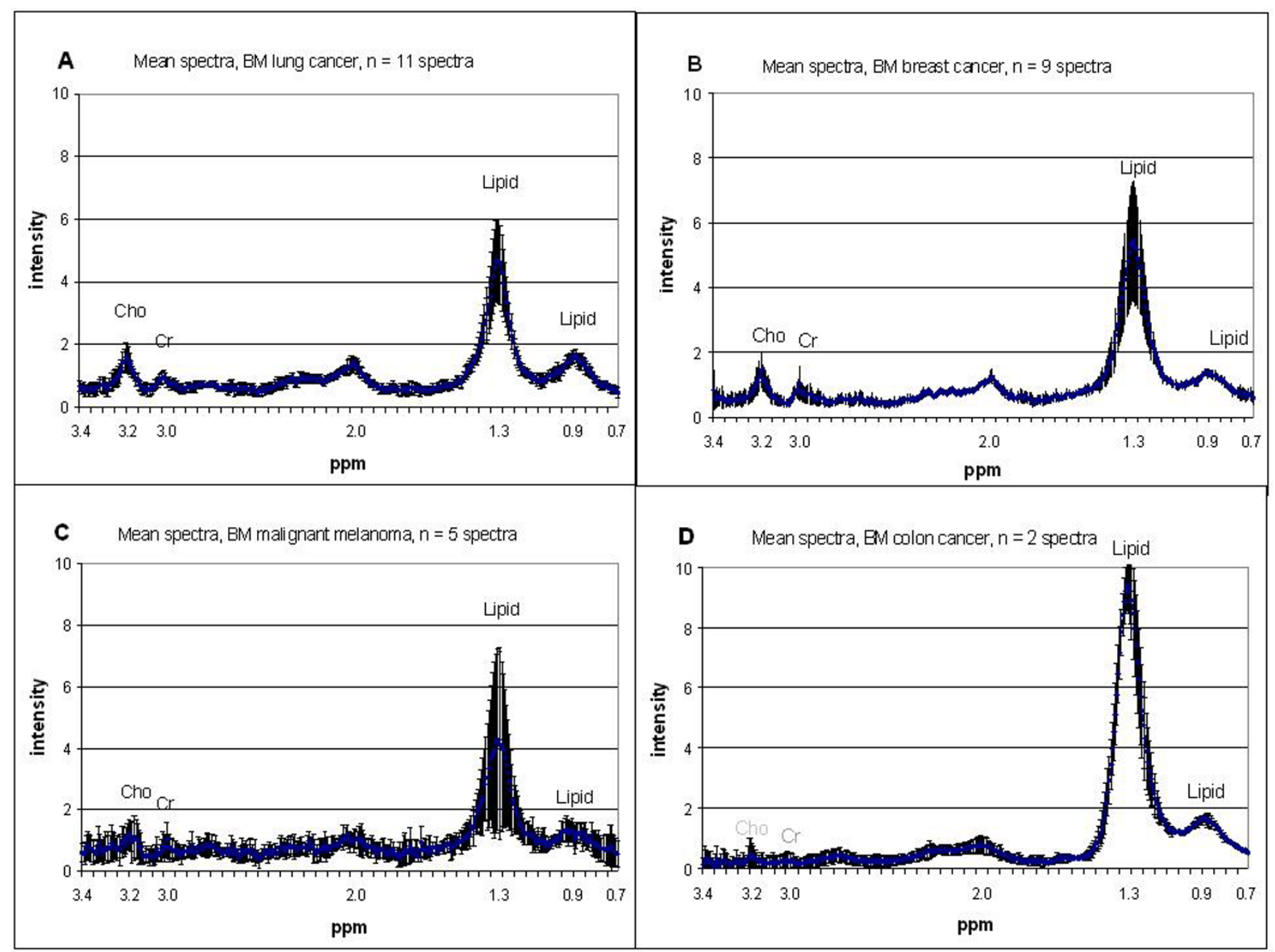

Figure 2

Mean spectra. Short echo time mean spectra $\pm 95 \% \mathrm{Cl}$ of brain metastases from different primary cancer. A: lung cancer (n = II spectra), B: breast cancer ( $n=9$ spectra), C: malignant melanoma $(n=5$ spectra), D: colon cancer $(n=2$ spectra). The 0.7 - 3.4 Ppm area of the spectra and the detected metabolites (in Ppm) are given; tCho (3.2), $\mathrm{Cr}$ (3.0): creatine and lipids (I.3, 0.9): methylene and methyl groups. 
up spectra are shown in Fig. 5. At the last examination four of the seven obtained spectra (four patients) showed decreased levels of the lipid signal (30-80\% peak intensity of mean normalized spectra). Of the ten second examination spectra six showed minor changes in the metabolite distribution, while three spectra indicated an increase in the $1.3 \mathrm{ppm}$ lipid peak relative to the signal of methyl at $0.9 \mathrm{ppm}$. The tenth spectra, obtained from the patient treated with chemotherapy showed an increasing signal of NAA and Cr peak relative to Cho-containing signals at $3.2 \mathrm{ppm}$ indicating normal brain tissue enclosed in the VOI due to the shrinking metastasis during the treatment period.

\section{Discussion}

The results presented here demonstrate that the lipid signals are important in brain metastases characterization.
The clustering of spectra from brain metastases from breast and lung cancer in the PCA score plot is described by PC1 showing the lipid signal as the dominating difference. The lipid signals are also an important factor in the PLS relating MR spectra to clinical outcome. These signals change after radiation therapy, which is in accordance to previous studies $[14,16]$. Many cellular processes such as proliferation, inflammation, malignancy, necrosis, growth arrest and apoptosis, have been linked to alterations in MR visible lipid levels. Intracellular lipid bodies appear to be the most important contributors [20,21]. However, which biochemical processes that lead to lipid accumulation of MR visible signals is still enigmatic, and the source and complete characteristics of the lipids found mostly in high grade brain tumors are still not elucidated $[21,22]$.

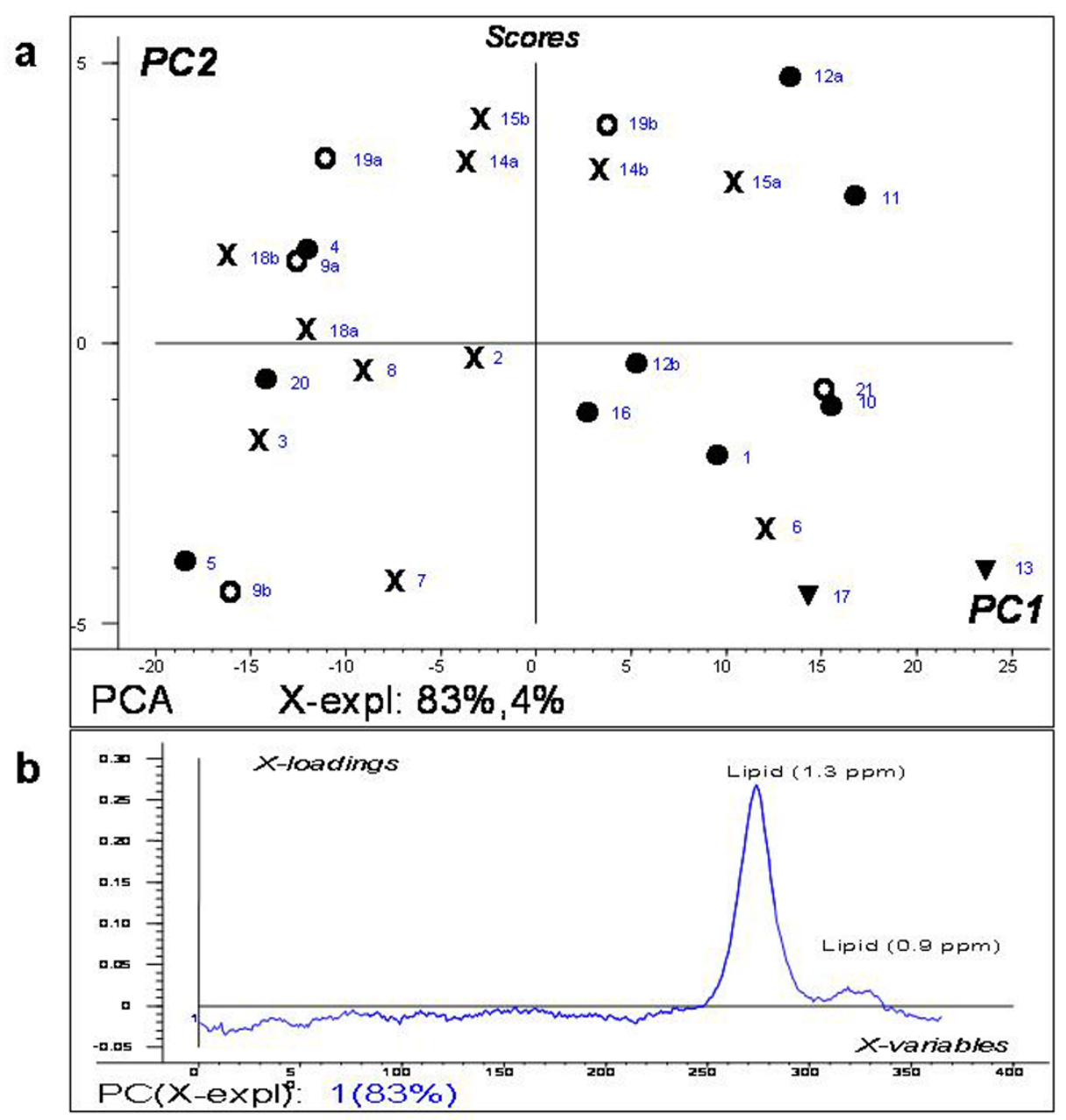

Figure 3

PCA results. Part a: Score plot of $\mathrm{PCl}$ versus $\mathrm{PC2}$ of water suppressed in vivo spectra. $0=$ breast cancer $(\mathrm{n}=9)$, $\mathbf{X}=$ lung cancer $(n=I I), \mathbf{O}=$ malignant melanoma $(n=5), \boldsymbol{\nabla}=$ colon cancer $(n=2)$. Part b: The loading profile of PCI showing differences in the lipid signals. 


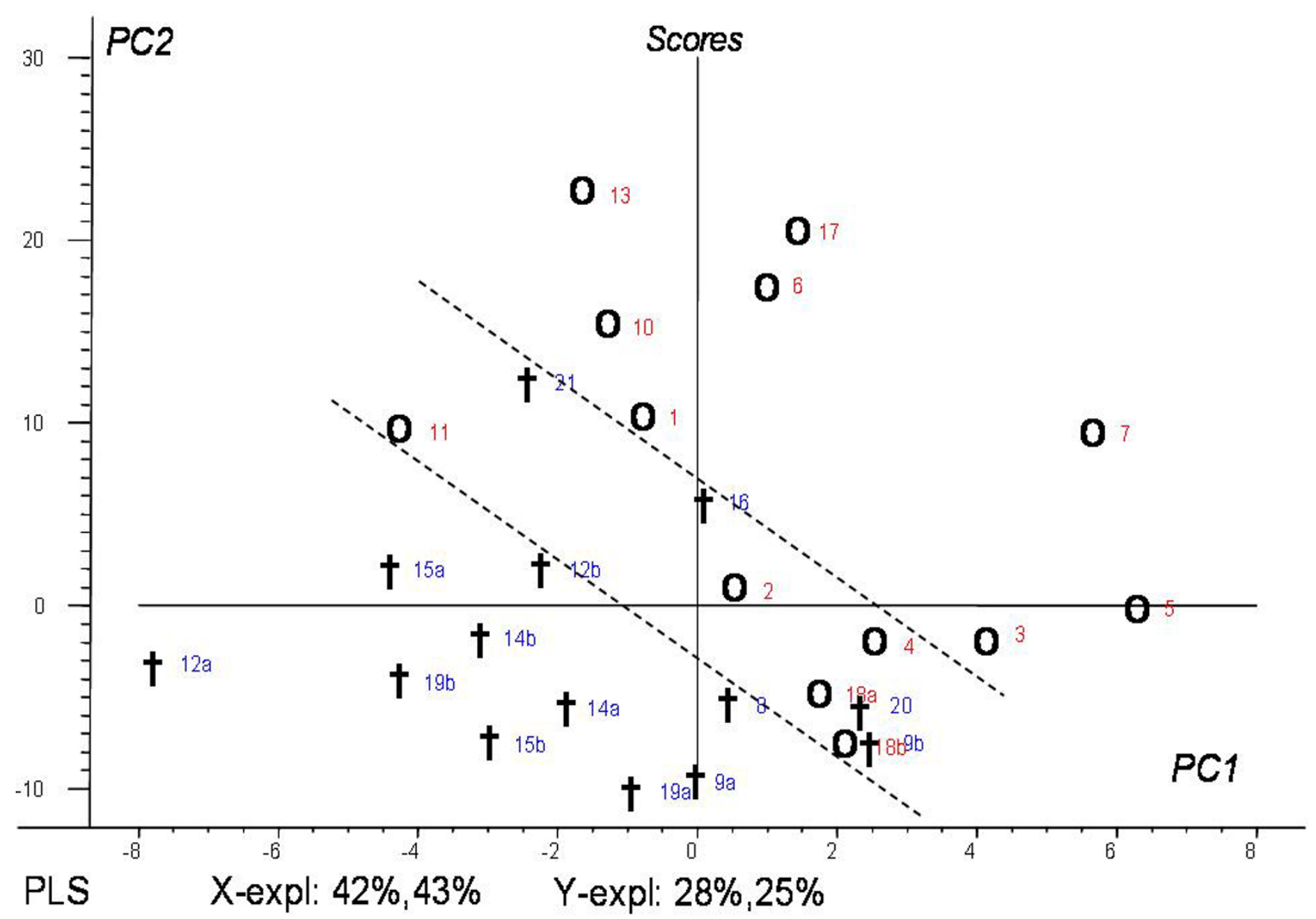

\section{Figure 4}

PLS score plot. Score plot of spectra from patients who lived longer than five months ( $\bigcirc)$, and they who passed away before five months after the first MR spectra examination $(t)$. The numbers refers to patient numbers in Table I. Some patients were examined for two metastases ( $a$ and $b)$.

Small signals of intracellular lipids might be observed in normal brain tissue in short echo time spectra and these signals are often increased in malignancies. In accordance with previous studies of brain tumors $[8,14]$, increased signals of lipids and also tCho compounds were detected in the majority of all spectra of brain metastases obtained with short echo time in this study (Fig. 2). The other main metabolites in spectra from normal brain tissue, NAA and $\mathrm{Cr}$ were definitely reduced in these spectra. The high intensity lipid signals and low levels of $\mathrm{Cr}$ are typical for metastases and have been used to differentiate these lesions from primary brain tumors [22-25]. However, the spectral differences between metastases and high grade gliomas are small and these two groups might be hard to distinguish [23]. Methylene components in fatty acids give rise to a broad peak around 2.0 - $2.2 \mathrm{ppm}$ [21] and this might overlap any possible NAA present. At longer echo time the lipid signals will be reduced due to short relaxation time [26], and in seven of the fourteen spectra obtained at TE 144, a small peak of NAA could be observed. Previous studies have interpreted that signal from NAA in metastases is due to the presence of viable neurons within an infiltrative tumour or contamination from normal brain tissue since NAA is a neuron specific metabolite [24,27].

The use of multivariate analyses can reveal features in the spectra which may not be detectable with traditional statistical analyses of peak integral ratios $[28,29]$. The mean spectra from the different primary cancer groups showed minor differences in distribution of the metabolites. Still, the spectra from metastases in breast and lung cancer patients tend to cluster into different parts of the PCA score plot, the separation being mainly due to differences 


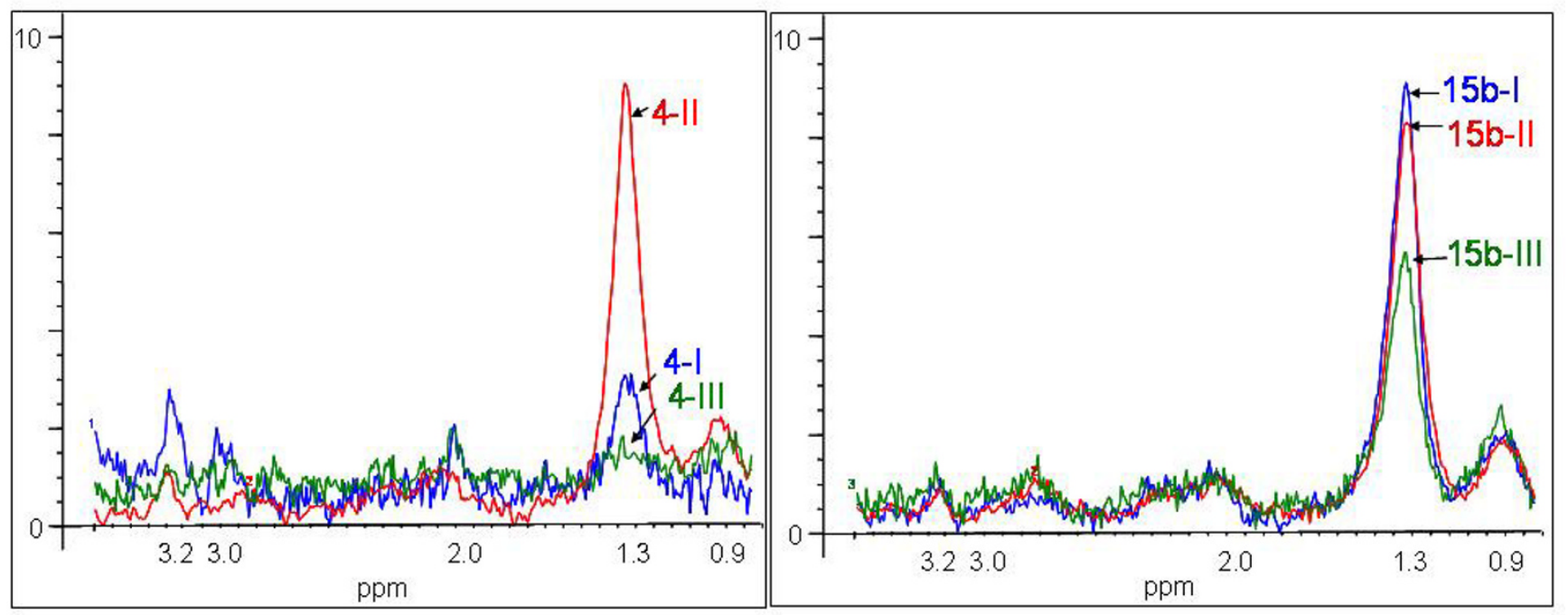

\section{Figure 5}

Follow-up spectra. Spectra of brain metastases from patients with primary breast (patient 4) and lung cancer (patient 15), before (-I), immediately after (-II) and two months after end of radiation treatment (-III). The patients' survival was more than 16 months and only 3 months, respectively.

in the lipid signals as described by PC1 (Fig. 3b). The dispersion of the malignant melanoma spectra in the PCA score plot (Fig. 3a) reflects the large confidence interval for the corresponding averaged spectra (Fig. 2c).

In this study, PLS was used to relate spectral data to clinical outcome for each patient. A trend of two separate clusters of patients surviving five months versus patients passing away before five months can be observed in the score plot (Fig. 4) The loading weights from PLS indicate that resonances in the ppm regions $0.8-0.9,1.8-2.2$ and $2.5-2.8 \mathrm{ppm}$ representing different lipid signals are most important for the prediction. By seeing the patients' performance status in conjunction with the respective localization in the score plot (Fig. 4), patients classified as RPA2 and 3 are dispersed across the plot, while the RPA1 classified patients are clustered in the intermediate area. Patients classified as RPA3 might be interpreted to have a better prognosis if the spectral data appear to be in the survival area of the score plot. Thus, the use of multivariate analysis on the spectral data might be of importance to predict the outcome for patients with brain metastases.

Half of the patients in this study received radiation therapy and the follow-up spectroscopy examinations were done immediately after completed treatment. However, only a few of these patients were able to fulfil the study protocol due to their health condition. The changes in level of mobile lipids and choline observed in spectra obtained after completed radiation therapy could reflect effects of the treatment observed at a metabolic level. Graves et al. [30] have previously shown changes in the metabolic profile of recurrent gliomas up to 14 months after gamma knife surgery. They observed an increase in the lactate/lipid resonance that developed early after the treatment but was reduced later in the follow-up of these glioma patients. Nelson [31] reported that the intensity of the tCho, Cr, and NAA signal decreased with time after radiation treatment of gliomas, and the lactate/lipid ratio increased to a maximum four months after the end of radiation. This is interpreted to represent a reduction in tumor and formation of treatment-induced necrosis. Possibly, the changes observed in the present study of brain metastases could be caused by the same mechanisms.

Single voxel spectroscopy has been extensively used in examination of brain tumors $[8,22,24,26]$ and has been included in routine MRI protocols for tumor diagnostics at several sites. Differentiation of most tumor types has been successfully reported and a clinical decision support system for classification of brain tumors, INTERPRET, has recently been developed [32]. The short acquisition time makes it simple to include it in a clinical MRI exam and the $3 \mathrm{~T}$ generation of MR instruments will give better spec- 
tral resolution and signal-to-noise ratio with equal effective size of VOI [12]. The spatial resolution will, however, be poor compared to MR Spectroscopic Imaging (MRSI). Using MRSI, several single voxels over a wider area become examined at the same time and metabolite distribution in both tumor and surrounding normal tissue are explored. However, single voxel MRS is less time consuming both during optimization and acquisition than MRSI. The signal from the whole metastasis was used for studying the metabolic phenotype in these analyses.

The exclusion criterion used in this study was the mean FWHM value for the corresponding unsuppressed water signal of the obtained spectra. This threshold value should not be considered as an exact threshold normative for other studies due to the small group investigated. However, the method of excluding spectra that are outside the $99 \%$ confidence interval of the data could be a used as a standardized quality control. The exclusion criterion excluded five of the enrolled twenty-six patients. Due to their bad health condition, some of the patients where uncomfortable during the examination which could cause motion artefacts, while in three of the patients the metastases were localized near the skull or close to cerebellum. The use of contrast agent has shown no significant effect on metabolite signal intensities at $1.5 \mathrm{~T}[33,34]$. The effect of the contrast agent has not been tested in this study. However, for clinical MRS studies of brain metastases in vivo the contrast agent will always be present as a part of the routine MRI protocol.

Spectrum quality also depends on the voxel size. The spectral SNR increases linearly with the voxel size and with the square root of the number of acquisitions [35]. To obtain maximum SNR within acceptable examination time, the number of acquisitions used was chosen as many as possible, resulting in 192 acquisitions during a seven minutes examination. In about half of the patient group, the smallest possible voxel size was used due to the size of the metastasis. Hence, these cases should give reduced SNR compared to the other cases where the cubic sides where $15 \mathrm{~mm}$. Among the excluded spectra two out of five where obtained using the largest volume size.

A clear limitation of the present study is the small and heterogeneous group of patients and increased number of patients to further validate the clinical value of the PLS model is necessary. A possible bias could be the skewed distribution of the subgroups of brain metastases. There were twice as many patients with brain metastases from breast and lung cancer as the other groups. This reflects however the incidence of brain metastases from the different type of primary cancer [1].

\section{Conclusion}

The in vivo metabolic MR profiles of brain metastases demonstrated variations due to origin of primary cancer and as an effect of radiation treatment. A correlation between MR profiles and survival at five months was also found. Thus, MR determined metabolic profiles might contain valuable clinical information for planning and evaluation of brain metastasis treatment.

\section{Competing interests}

The author(s) declare that they have no competing interests.

\section{Authors' contributions}

The study was conceived by ISG, SL and US while all authors contributed to the design of it. SL and RJ included the patients and provided the clinical information. TES organized the MRS examinations, performed the data analyses and wrote the paper. KAK were responsible for the MRI and MRS examinations. TFB contributed with multivariate analyses and discussions. All authors discussed, read and approved the final manuscript.

\section{Acknowledgements}

This work was supported by a grant from Central Norway Regional Health Authority. Thanks to the radiographs at Department of Radiology, St. Olavs University Hospital, for great technical assistance during the MRI/MRS examinations.

\section{References}

I. Sawaya R, Bindal RK, Lang FF, Abi-Said D: Metastatic brain tumors. In Brain Tumors An encyclopedic approach Edited by: Kaye $\mathrm{AH}$, Laws ER Jr. London: Harcourt Publishers Limited; 200I:999-1026.

2. Barnholtz-Sloan JS, Sloan AE, Davis FG, Vigneau FD, Lai P, Sawaya RE: Incidence proportions of brain metastases in patients diagnosed (1973 to 200I) in the Metropolitan Detroit Cancer Surveillance System. J Clin Oncol 2004, 22:2865-2872.

3. Gaspar LE, Scott C, Murray K, Curran W: Validation of the RTOG recursive partitioning analysis (RPA) classification for brain metastases. Int J Radiat Oncol Biol Phys 2000, 47:I00I-I006.

4. Kaal EC, Niel CG, Vecht CJ: Therapeutic management of brain metastasis. Lancet Neurol 2005, 4:289-298.

5. Patchell RA: The management of brain metastases. Cancer Treat Rev 2003, 29:533-540.

6. Salvati M, Cervoni L, Raco A: Single brain metastases from unknown primary malignancies in CT-era. J Neurooncol 1995, 23:75-80.

7. Golder W: Magnetic resonance spectroscopy in clinical oncology. Onkologie 2004, 27:304-309.

8. Howe FA, Barton SJ, Cudlip SA, Stubbs M, Saunders DE, Murphy M, Wilkins P, Opstad KS, Doyle VL, McLean MA, Bell BA, Griffiths JR: Metabolic profiles of human brain tumors using quantitative in vivo I H magnetic resonance spectroscopy. Magn Reson Med 2003, 49:223-232.

9. Negendank WG, Sauter R, Brown TR, Evelhoch JL, Falini A, Gotsis ED, Heerschap A, Kamada K, Lee BC, Mengeot MM, Moser E, Padavic-Shaller KA, Sanders JA, Spraggins TA, Stillman AE, Terwey B, Vogl TJ, Wicklow K, Zimmerman RA: Proton magnetic resonance spectroscopy in patients with glial tumors: a multicenter study. J Neurosurg 1996, 84:449-458.

10. Barker PB, Hearshen DO, Boska MD: Single-voxel proton MRS of the human brain at I.5T and 3.0T. Magn Reson Med 200I, 45:765-769.

II. Gonen O, Gruber S, Li BS, Mlynarik V, Moser E: Multivoxel 3D proton spectroscopy in the brain at $\mathrm{I} .5$ versus $3.0 \mathrm{~T}$ : signal-to- 
noise ratio and resolution comparison. Am J Neuroradiol 200I, 22:|727-|73|.

12. Sjobakk TE, Lundgren S, Kristoffersen A, Singstad T, Svarliaunet AJ, Sonnewald U, Gribbestad IS: Clinical IH magnetic resonance spectroscopy of brain metastases at I.5T and 3T. Acta Radiol 2006, 47:501-508.

13. Frahm J, Bruhn H, Hanicke W, Merboldt KD, Mursch K, Markakis E: Localized proton NMR spectroscopy of brain tumors using short-echo time STEAM sequences. J Comput Assist Tomogr 1991, I 5:915-922.

14. Nelson SJ: Multivoxel magnetic resonance spectroscopy of brain tumors. Mol Cancer Ther 2003, 2:497-507.

15. Sijens PE, Vecht CJ, Levendag PC, van DP, Oudkerk M: Hydrogen magnetic resonance spectroscopy follow-up after radiation therapy of human brain cancer. Unexpected inverse correlation between the changes in tumor choline level and postgadolinium magnetic resonance imaging contrast. Invest Radiol 1995, 30:738-744.

16. Weybright P, Sundgren PC, Maly P, Hassan DG, Nan B, Rohrer S, Junck L: Differentiation between brain tumor recurrence and radiation injury using MR spectroscopy. AJR Am J Roentgenol 2005, I 85: | $47 \mid-1476$.

17. Li X, Jin H, Lu Y, Oh J, Chang S, Nelson SJ: Identification of MRI and IH MRSI parameters that may predict survival for patients with malignant gliomas. NMR Biomed 2004, 17:10-20.

18. Naressi A, Couturier C, Castang I, de Beer R, Graveron-Demilly D: Java-based graphical user interface for MRUI, a software package for quantitation of in vivo/medical magnetic resonance spectroscopy signals. Comput Biol Med 200 I, 3 I:269-286.

19. Kennard RW, Stone LA: Computer aided design of experiments. Techometrics 1969, II:137-148.

20. Barba I, Cabanas ME, Arus C: The relationship between nuclear magnetic resonance-visible lipids, lipid droplets, and cell proliferation in cultured C6 cells. Cancer Res 1999, 59:186|-|868.

21. Hakumaki JM, Kauppinen RA: IH NMR visible lipids in the life and death of cells. Trends Biochem Sci 2000, 25:357-362.

22. Opstad KS, Murphy MM, Wilkins PR, Bell BA, Griffiths JR, Howe FA: Differentiation of metastases from high-grade gliomas using short echo time IH spectroscopy. J Magn Reson Imaging 2004, 20:187-192.

23. Hollingworth W, Medina LS, Lenkinski RE, Shibata DK, Bernal B, Zurakowski D, Comstock B, Jarvik JG: A systematic literature review of magnetic resonance spectroscopy for the characterization of brain tumors. AJNR Am J Neuroradiol 2006, 27:|404-|4||.

24. Ishimaru H, Morikawa M, Iwanaga S, Kaminogo M, Ochi M, Hayashi K: Differentiation between high-grade glioma and metastatic brain tumor using single-voxel proton MR spectroscopy. Eur Radiol 200I, II:I784-1791.

25. Majos C, Alonso J, Aguilera C, Serrallonga M, Perez-Martin J, Acebes JJ, Arus C, Gili J: Proton magnetic resonance spectroscopy ((I)H MRS) of human brain tumours: assessment of differences between tumour types and its applicability in brain tumour categorization. Eur Radiol 2003, I3:582-59|.

26. Majos C, Julia-Sape M, Alonso J, Serrallonga M, Aguilera C, Acebes JJ, Arus C, Gili J: Brain tumor classification by proton MR spectroscopy: comparison of diagnostic accuracy at short and long TE. AJNR Am J Neuroradiol 2004, 25:1696-1704.

27. Kinoshita $Y$, Kajiwara $H$, Yokota A, Koga $Y$ : Proton magnetic resonance spectroscopy of brain tumors: an in vitro study. Neurosurgery 1994, 35:606-613.

28. Lukas L, Devos A, Suykens JA, Vanhamme L, Howe FA, Majos C, Moreno-Torres A, van der GM, Tate AR, Arus C, Van Huffel S: Brain tumor classification based on long echo proton MRS signals. Artif Intell Med 2004, 31:73-89.

29. Bathen TF, Sjobakk TE, Skranes J, Brubakk AM, Vik T, Martinussen M, Myhr GE, Gribbestad IS, Axelson D: Cerebral metabolite differences in adolescents with low birth weight: assessment with in vivo proton MR spectroscopy. Pediatr Radiol 2006, 36:802-809.

30. Graves EE, Nelson SJ, Vigneron DB, Verhey L, McDermott M, Larson D, Chang S, Prados MD, Dillon WP: Serial proton MR spectroscopic imaging of recurrent malignant gliomas after gamma knife radiosurgery. AJNR Am I Neuroradiol 200I, 22:6I3-624.

31. Nelson SJ, Graves E, Pirzkall A, Li X, Antiniw CA, Vigneron DB, McKnight TR: In vivo molecular imaging for planning radiation therapy of gliomas: an application of IH MRSI. J Magn Reson Imaging 2002, 16:464-476.

32. Tate AR, Underwood J, Acosta DM, Julia-Sape M, Majos C, MorenoTorres A, Howe FA, van der GM, Lefournier V, Murphy MM, Loosemore A, Ladroue C, Wesseling P, Luc BJ, Cabanas ME, Simonetti AW, Gajewicz W, Calvar J, Capdevila A, Wilkins PR, Bell BA, Remy C, Heerschap A, Watson D, Griffiths JR, Arus C: Development of a decision support system for diagnosis and grading of brain tumours using in vivo magnetic resonance single voxel spectra. NMR Biomed 2006, 19:4| I-434.

33. Sijens PE, Oudkerk M, van Dijk P, Levendag PC, Vecht CJ: I H MR spectroscopy monitoring of changes in choline peak area and line shape after Gd-contrast administration. Magn Reson Imaging 1998, 16:1273-1280.

34. Smith JK, Kwock L, Castillo M: Effects of contrast material on single-volume proton MR spectroscopy. AJNR Am J Neuroradiol 2000, 2 I: I084-1089.

35. Haacke EM, Brown RW, Thompson MR, Venkatesan R: Signal, Contrast and Noise. In Magnetic Resonance Imaging: Physical Principles and Sequence Design Edited by: Haacke EM, Brown RW, Thompson MR, Venkatesan R. New York: Wiley-Liss; 1999:33 I-380.

\section{Pre-publication history}

The pre-publication history for this paper can be accessed here:

http://www.biomedcentral.com/1471-2407/7/141/pre pub
Publish with Bio Med Central and every scientist can read your work free of charge

"BioMed Central will be the most significant development for disseminating the results of biomedical research in our lifetime. "

Sir Paul Nurse, Cancer Research UK

Your research papers will be:

- available free of charge to the entire biomedical community

- peer reviewed and published immediately upon acceptance

- cited in PubMed and archived on PubMed Central

- yours - you keep the copyright 